\title{
Tumor Suppressive Functions of p53
}

\author{
Jack T. Zilfou ${ }^{1}$ and Scott W. Lowe ${ }^{2,3}$ \\ ${ }^{1}$ Zilfou Therapeutics, Inc., Allentown, Pennsylvania 18104 \\ ${ }^{2}$ Cold Spring Harbor Laboratory, Cold Spring Harbor, New York 11724 \\ ${ }^{3}$ Howard Hughes Medical Institute, Cold Spring Harbor, New York 11724 \\ Correspondence: jackzilfou@verizon.net
}

The majority of human cancers acquire mutations that abrogate the p53 tumor suppressor network and, as a consequence, p53 is one of the most extensively studied proteins in cancer research. Because of its potent tumor suppressive activity, it is widely assumed that a molecular understanding of p53 action will produce fundamental insights into natural processes that limit tumorigenesis and may identify key molecular targets for therapeutic intervention. p53 functions largely as a transcription factor, and can trigger a variety of antiproliferative programs by activating or repressing key effector genes. Despite a significant body of literature detailing the biochemical and biological functions of p53, much remains to be elucidated. Indeed, the p53 network is as complex and enigmatic as it is relevant. It is the goal of this article, written 30 years after the discovery of p53, to present a concise review of the tumor suppressor role of the p53 network and to highlight the context-dependent nature of p53 target-gene functions.

$\mathrm{D}$ ubbed as the "guardian of the genome" (Lane 1992) and the "cellular gatekeeper" (Levine 1997), the p53 protein acts to transmit a variety of stress-inducing signals to different antiproliferative cellular responses. Hence, p53 can be activated in response to DNA damage, oncogene activation, or hypoxia, in which it subsequently orchestrates biological outputs such as apoptosis, cell-cycle arrest, senescence, or modulation of autophagy (Yee and Vousden 2005; Riley et al. 2008; Green and Kroemer 2009). Importantly, these p53 tumor suppressor functions are context-dependent and may be influenced by numerous factors, including cell type, microenvironment, and oncogenic events acquired during the course of tumor evolution. This suggests that, although expression of many genes in the p53 network may be altered in tumors, the critical nodes of the network may differ based on the tumor-cell context. The challenge is to understand which p53 targets or functions are key to the evolution of different tumor types, as this may ultimately identify activities for tumor maintenance and suggest targets for therapeutic intervention.

\section{MODELS FOR p53 ACTIVATION}

The traditional view describing p53 activation in response to cellular stress comprises three

Editors: Arnold J. Levine and David Lane

Additional Perspectives on The p53 Family available at www.cshperspectives.org

Copyright (C) 2009 Cold Spring Harbor Laboratory Press; all rights reserved; doi: 10.1101/cshperspect.a001883

Cite this article as Cold Spring Harb Perspect Biol 2009;00:a001883 
basic steps: stabilization of $\mathrm{p} 53$, sequencespecific DNA binding, and transcriptional activation of target genes (Yee and Vousden 2005). p53 stabilization is primarily achieved through events that disrupt its interaction with $\mathrm{Mdm} 2$, a negative regulator that mediates a ubiquitinmediated degradation of p53. For example, in response to DNA damage from ionizing radiation or certain chemotherapeutic agents, p53 is posttranslationally modified, including phosphorylation of the amino terminus of $\mathrm{p} 53$ at specific amino acids, by various kinases, including ATM, ATR, DNA-PK, Chk1, and Chk2 (Appella and Anderson 2001). The amino-terminal phosphorylation of p53 prevents Mdm2 binding, resulting in the stabilization of p53. p53 stabilization also occurs in response to oncogenic challenges to the cell, although this response is primarily mediated through the antagonism of the p53-Mdm2 interaction by the tumor suppressor $\mathrm{p} 14^{\mathrm{ARF}}$ ( $\mathrm{p} 19^{\mathrm{ARF}}$ in the mouse) (Sherr 2006).

Following its stabilization, p53 binds DNA in a sequence-specific manner (el-Deiry et al. 1992). The DNA-binding domain of the p53 protein is a "hot spot" for mutation, as the majority of tumor-associated mutations in p53 occur within this region (Hainaut and Hollstein 2000). This finding underscores the importance of p53 binding to DNA in a sequence-specific manner. p53 also contains a carboxy-terminal basic DNA binding domain, originally shown to inhibit p53 binding to sequence-specific DNA in in vitro assays, that facilitates structure-specific p53 DNA binding in in vivo chromatin immunoprecipitation (ChIP) analysis. However, the simple model of stress-induced, sequence-specific DNA binding is challenged by the recent findings that a significant portion of $\mathrm{p} 53$ is bound to DNA in unstressed cells via the carboxyterminal domain (Liu et al. 2004b).

One rationale for the ubiquitous DNA binding activity of the carboxy-terminal domain of p53 is to facilitate DNA binding and the search for p53 target sites following cellular stress. In addition, studies suggest that basal p53 levels are critical for the assembly of the preinitiation complex on the p21 promoter in unstressed cells (Espinosa et al. 2003). Taken together, these studies support the idea that p53 can control gene expression in stressed and unstressed cells.

After its stabilization and sequence-specific DNA binding, p53 activates or represses its target genes. The traditional view suggests that p53 promotes transcriptional activation or repression of target genes by interacting with general transcriptional factors such as TFIID/ TAFs. However, such a model under-appreciates the complexity of promoter selection. Promoter selection is dictated by numerous factors, including posttranslational modifications of p53 that can influence the recruitment of p53 binding proteins to specific promoters. For example, p53 can interact with various transcriptional activators, such as histone acetyltransferase CBP/p300 (Iyer et al. 2004), and transcriptional repressors and corepressors, such as histone deacetylases and $\sin 3$ (Zilfou et al. 2001; Murphy 2003), to modulate transcription. The CBP/ p300-p53 interaction facilitates both histone and p53 acetylation, leading to a more open chromatin conformation near p53 targets and a more active p53 protein, respectively (Chan and La Thangue 2001).

A recent review addressing the modes of p53 regulation proposed an under-appreciated step in the physiological activation of p53 (Kruse and $\mathrm{Gu}$ 2009). The authors synthesized the voluminous data on the regulation of p53 and concluded that p53 activation in vivo requires not only its stabilization and activation, but also the release of p53 from an actively repressed state. This step, dubbed "antirepression," is rooted in studies implying that p53 is intrinsically active, but repressed by its negative regulators Mdm2 and Mdmx (Montes de Oca Luna et al. 1995; de Rozieres et al. 2000; Parant et al. 2001).

This intrinsic potency of p53 is supported by several observations. First, although $m d m 2$ or $m d m x$ (aka, $m d m 4$ ) deficient mice die during embryonic development, this lethality is rescued by codeletion of p53 (Montes de Oca Luna et al. 1995; Parant et al. 2001). Second, Mdm2 inactivation in cultured cells triggers p53-mediated apoptosis (de Rozieres et al. 2000). Finally, mice harboring a knock-in allele of p53 that is 
defective in Mdm2 binding ( $\mathrm{p} 53 \mathrm{QS}$ ), but retains the ability to bind DNA and transactivate some p53 target genes, die during embryogenesis (Johnson et al. 2005). This embryonic lethality may be caused by the inability of $\mathrm{mdm} 2$ to interact with and repress the activity of the p53QS mutant.

Based on the previous discussion, Kruse and $\mathrm{Gu}$ (2009) proposed a refined three-step model for promoter-specific activation of p53 activation. The first step is the stress-induced stabilization of p53 that can occur through various mechanisms, many of which function by inhibiting the ability of $\mathrm{Mdm} 2$ to ubiquitinate and degrade p53. The second step is the "antirepression," or release of $\mathrm{p} 53$ from the $\mathrm{Mdm} 2$ and Mdmx mediated inhibition. This step requires the acetylation of p53 at specific amino-acid residues, and results in the select activation of a subset of p53 target genes. The third step is the full activation of specific promoters facilitated by the recruitment and interaction of numerous cofactors by p53. These cofactors can modify p53, histones, or other transcription factors. Still poorly understood are the precise mechanisms whereby p53 targets specific genes involved in apoptosis, senescence, cell-cycle arrest, or autophagy, although this may involve specific combinations of cofactors and posttranslational modifications. Indeed, combinations of p53 posttranslational modifications, including phosphorylation, ubiquitination, methylation, sumoylation, neddylation, and acetylation, may serve as a discrete "barcode," enabling p53 to activate specific promoters (Kruse and Gu 2009).

\section{p53 EFFECTOR FUNCTIONS}

Genetic studies in cultured cells and mice have implicated p53 in a variety of antiproliferative functions, each of which, under the appropriate circumstances, may contribute to its tumor suppressive properties. Some of the most important of these are discussed in the following sections.

\section{Cell-cycle Checkpoints}

Cell-cycle checkpoints are important control features that ensure the fidelity of cell division by verifying whether the processes at each phase of the cell cycle have been accurately completed before progression into the next phase. In response to various cellular stresses, cells may undergo growth arrest at these checkpoints to prevent the propagation of mutations in the DNA. The role of p53 in cellular growth arrest has been extensively studied, and many of the key upstream regulators and downstream effectors of p53 have been described (Giono and Manfredi 2006). For example, murine embryonic fibroblasts (MEFs) exposed to DNA damage activate the ATM/ATR pathways, leading to the activation of p53 and subsequently undergo G1 arrest. p53 induces a G1 arrest primarily through the transactivation of $p 21^{\text {Wafl/Cip } 1}$, a cyclin-dependent kinase inhibitor. Targeted disruption of the $p 21^{\text {Waf } 1 / C i p 1}$ gene has been shown to compromise the G1/S checkpoint in MEFs and significantly extend their lifespan (Brugarolas et al. 1995; Deng et al. 1995). However, the effects are not as drastic as p53 loss, suggesting that other p53 effectors contribute to this process.

Progression of cells from G2 to mitosis is driven by the maturation-promoting factor (MPF), which comprises a complex of cyclin B1 and cdc2. p53 has been shown to induce G2/M arrest by primarily perturbing the function of the cyclin B1/cdc2 complex. Specifically, p53 represses $\operatorname{cdc} 25 \mathrm{c}$, a phosphatase that promotes mitosis, after DNA damage (St Clair and Manfredi 2006). Additionally, p53 has been shown to transcriptionally activate $14-3-3 \sigma$ after DNA damage (Hermeking et al. 1997). 14-3-3o is a protein that prevents proper nuclear localization of cyclin B1/cdc2 after DNA damage. Moreover, deletion of 14-3-3 $\sigma$ in HCT116 cells resulted in cell death in response to DNA damage (Chan et al. 1999).

\section{Cellular Senescence}

Cellular senescence is a permanent form of cellcycle arrest that was originally described in normal human fibroblasts by Hayflick (Hayflick 1965). Two important hypotheses eventually emerged to describe the biological importance of senescence in vivo; one proposing that senescence is beneficial to the organism by 
functioning as a tumor-suppressive mechanism, whereas the other suggested that senescence is deleterious to the organism as it recapitulated the aging or loss of regenerative capacity of cells (Campisi and d'Adda di Fagagna 2007). Senescence can be induced by various stressors, including dysfunctional telomeres, nontelomeric DNA damage, excessive mitogenic signaling (including those produced by oncogenes), and perturbations in chromatin organization. DNA tumor virus oncoproteins can bypass senescence, and this frequently involves their ability to inactivate p53. Importantly, in addition to p53, the RB tumor suppressor pathway also contributes to senescence (Stewart and Weinberg 2006).

The senescence response involves significant changes in cellular phenotypes. For example, senescent cells have a large and flattened morphology, and show marked but consistent changes in their gene expression profile, consistent with a possible role in wound healing (Krizhanovsky et al. 2008a). They are unable to replicate DNA and, consequently, undergo permanent cell-cycle arrest. Several markers are typically used to identify senescent cells, albeit no markers currently in use are exclusive to the senescent state. These include the senescenceassociated $\beta$-galactosidase (SA- $\beta$ gal), as well as increased expression of the p16INK4a and p53INK4b cyclin-dependent kinase inhibitors (Campisi and d'Adda di Fagagna 2007).

The permanent cell-cycle arrest program that accompanies senescence is considered most pertinent to its putative tumor suppressive role. Interestingly, because certain oncogenes can trigger senescence, mutations that bypass senescence, for example in the p53 network, cooperate with these changes to promote full malignant transformation (Serrano et al. 1997). Many chemotherapeutic agents that cause DNA damage and activate p53 can also induce senescence, and indeed, disruption of p53 can disable drug-induced senescence and lead to tumor progression (Schmitt et al. 2002b; Roninson 2003).

As previously noted, the senescence program can be established and maintained by the p53 and p16-RB tumor-suppressor pathways.
These pathways can respond to similar or different stimuli, and can show cell-type specificity with respect to their ability to induce senescence. Moreover, although these pathways have been shown to independently inhibit cell-cycle progression, there is notable cross talk among them. For example, p14ARF can detect various senescence signals, and by binding $\mathrm{Mdm} 2$, can activate p53. p53 can subsequently transactivate $p 21^{\text {Waf1/Cip1 }}$, which, in this context, contributes to p53-dependent senescence rather than a reversible checkpoint arrest (Brown et al. 1997). p21 Waf1/Cip1 can also inhibit cyclin-dependent kinases upstream of the RB tumor suppressor. $\mathrm{RB}$ can in turn inhibit E2F, a potent inducer of cell proliferation. Per the RB pathway, deregulation of E2F can activate p14ARF, and subsequently lead to p53 activation. The cross talk and redundancy between the p53 and p16-RB pathways underscores the importance of senescence as a tumor suppressive biological output. Moreover, this redundancy highlights the complexity and interplay among the various stimuli, cell types, and genetic backgrounds of cells destined for senescence.

It is unclear precisely how the p53 and p16$\mathrm{RB}$ pathways establish and maintain senescence. Both of these pathways can induce transient cell-cycle arrest, and presumably are modified by senescence signals. The relevant query is what dictates whether cells undergo transient (checkpoint arrest) or irreversible cell-cycle arrest (senescence). It may depend on whether or not the senescence signal is sustained, or possibly the cooperation of secondary events that "lock" the arrest into an irreversible state. In this regard, it was found that RB directs the formation of SAHFs, senescence-associated heterochromatin foci that contain and are thought to stably silence E2F target genes (Narita et al. 2003; Narita et al. 2006).

Recent studies of senescence show that some of the p53 tumor suppressor activity is noncell autonomous. Specifically, among the numerous transcriptional changes in senescent cells are increases in various secreted factors, thus providing the basis for the senescence-associated secretory phenotype. These changes involve the up-regulation of extracellular matrix (ECM) 
degrading enzymes, down-regulation of ECM production, and an overall up-regulation of immune modulating cytokines and chemokines. p53 plays a significant role in this process, as some of these molecules can be induced by $\mathrm{p} 53$ and are direct transcriptional targets (Gorgoulis et al. 2003; Xue et al. 2007).

The ability of p53 to modulate the senescence-associated secretory phenotype may contribute to its tumor-suppressive role. This was recently shown using RNA interference (RNAi) to conditionally regulate endogenous p53 expression in a mouse model of liver carcinoma (Xue et al. 2007). Reactivation of endogenous p53 in p53-deficient tumors produced complete tumor regression, and the primary cellular response was senescence. Importantly, this senescence program was associated with differentiation and the up-regulation of inflammatory cytokines, hence triggering an innate immune response that targeted tumor cells in vivo. These data illustrate how the cellular senescence program, driven by $\mathrm{p} 53$ in this setting, can act along with the innate immune system to significantly limit tumor growth. Additionally, if similar processes occur during the development of spontaneous tumors, then p53 would have a major impact on the immune surveillance of early lesions and its loss may contribute to immune evasion.

Most studies have focused on the role of senescence as a tumor suppressive mechanism. However, senescent cells have been observed in aged and damaged tissues, raising the possibility that the senescence program, and p53 action, may have broader roles. Recent studies of senescence have identified pathophysiologic roles for $\mathrm{p} 53$ beyond cancer. In one such study, a role for senescence in limiting liver fibrosis has been reported (Krizhanovsky et al. 2008b). Liver fibrosis is a tissue response to liver damage that can eventually produce cirrhosis and lead to hepatocellular carcinoma development. Senescent cells, primarily derived from activated hepatic stellate cells, accumulated in murine livers treated to produce fibrosis. Importantly, in mice lacking p53, stellate cells continue to proliferate, thus leading to excessive liver fibrosis. Additionally, senescent activated stellate cells show a gene expression profile consistent with a reduction in ECM components, enhanced secretion of ECM degrading enzymes, and enhanced immune surveillance. Consequently, the innate immune system, via natural killer cells, preferentially kills senescent activated stellate cells, thereby resolving the fibrosis (Krizhanovsky et al. 2008b).

The previous observations suggest that the senescence program can facilitate tissue homeostasis during certain wound healing responses, and identify the first noncancer pathology for which senescence plays a protective role. Additionally, these findings may aid in the elucidation of the mechanisms underlying druginduced liver injury, a common safety finding driving withdrawal of drugs from the market or preventing the approval of new drugs (Kaplowitz 2001).

\section{Autophagy}

Autophagy is a catabolic process involving the degradation of a cell's own components primarily through the lysosomal machinery. Autophagy can have both pro- and antioncogenic functions, which may reflect its action as either a prosurvival or prodeath mechanism (Eisenberg-Lerner and Kimchi 2009). Supporting an antioncogenic function, Beclin 1, a conserved protein that is essential for autophagy, is also a haploinsufficient tumor suppressor (Liang et al. 1999; Yue et al. 2003).

Recent tudies have implicated the p53 network in the modulation of autophagy. p53 has been shown to transcriptionally activate the damage-regulated autophagy modulator (DRAM) gene, producing a lysosomal protein, and induce autophagy in a DRAM-dependent manner (Crighton et al. 2006). DRAM has also been shown to be essential for p53-mediated apoptosis.

p53 has also been shown to inhibit autophagy. Specifically, inhibition of p53 in enucleated cells increases autophagy (Tasdemir et al. 2008). Moreover, expression of cytoplasmic, not nuclear, p53 repressed the enhanced autophagy in p53-null cells (Tasdemir et al. 2008). Here, the enhanced autophagy in p53-null cells is thought to confer a survival advantage to 
the tumor cell. This increase in autophagy may be explained, in part, by p14ARF, an important component of the p53 network. Loss of p53 can lead to significantly higher levels of p14ARF, and importantly, p14ARF can be a potent inducer of autophagy (Abida and $\mathrm{Gu}$ 2008; Humbey et al. 2008; Pimkina et al. 2009). Paradoxically, in this context, p14ARF may function as a prosurvival protein whose function is repressed by the presence of $\mathrm{p} 53$.

Based on current information, it appears that p53 imparts a complex mechanism of control on the autophagy program. The precise importance of the autophagy program to p53 biology remains unclear, but remains an actively pursued question. Recent studies have suggested that the induction of autophagy might facilitate the progression of apoptotic or senescence programs (Crighton et al. 2007; Young et al. 2009). In this view, p53 might coordinately activate autophagy with other effector programs to facilitate their successful completion.

\section{Apoptosis}

Although the ability of p53 to trigger cell-cycle arrest was discovered first, its action in controlling apoptosis is the most intensely studied. A p53-dependent apoptosis program was first noted following irradiation of mouse thymocytes (Clarke et al. 1993; Lowe et al. 1993). Shortly thereafter, it was shown that oncogenes could activate the p53 tumor suppressor leading to apoptosis, and that p53 is required for apoptosis induced by certain DNA damaging anticancer agents (Lowe and Ruley 1993). These studies revealed how $\mathrm{p} 53$ acts as part of an intrinsic fail-safe mechanism to resist transformation, and established a paradigm for understanding the genes and processes that determine the efficacy of cancer therapy.

Many more p53 target genes have been implicated in apoptosis than its other effector functions, suggesting that its control of the apoptotic program is complex. Current evidence indicates that the apoptotic activity of p53 is tightly controlled and is influenced by a series of quantitative and qualitative events that determine the outcome of p53 activation (Fridman and Lowe 2003). Along these lines, other p53 family members can induce apoptosis, either in concert or in parallel with p53. Apoptosis can be integrated into a larger p53 tumor suppressor network controlled by different signals, environmental factors, and cell type (Fridman and Lowe 2003).

Additionally, p53-mediated apoptosis involves the coordination of transcription-dependent and transcription-independent functions of p53. On activation, p53 can transactivate numerous genes involved in apoptosis, including Bax, PIG3, Killer/DR5, CD95 (Fas), p53AIP1, Perp, and BH3-only proteins Noxa and PUMA (p53-up-regulated modulator of apoptosis) (Riley et al. 2008). Many of these targets are members of the proapoptotic Bcl-2 family of genes.

In addition to these nuclear activities of p53, p53 also has cytosolic activities that can induce apoptosis in a transcription-independent manner (Green and Kroemer 2009). Specifically, in response to various cell death signals, such as ionizing radiation, p53 rapidly localizes to the mitochondria. At the mitochondria, p53 can induce mitochondrial outer membrane permeabilization (MOMP), thus leading to the release of pro-apoptotic factors from the mitochondrial intermembrane space. p53 can interact with $\mathrm{Bcl} 2, \mathrm{Bcl}-\mathrm{X}_{\mathrm{L}}$, and Bak at the mitochondria, and has been suggested to act like a BH3-only protein, either as a direct activator of Bax and/ or Bak, or as a derepressor.

The transcription-dependent and transcription-independent mechanisms of p53 have been recently linked through the p53 apoptotic target gene PUMA (Chipuk et al. 2005). Specifically, in response to cellular stress, p53 transactivates PUMA. PUMA then translocates to the mitochondria, where it can bind $\mathrm{Bcl}-\mathrm{X}_{\mathrm{L}}$ protein, thus releasing p53 to activate Bax. These data suggest that the transcription-dependent component of the p53 network is essential for the thorough induction of apoptosis, and PUMA plays a critical role in this process.

Indeed, PUMA is a unique p53 apoptotic target gene. It is the only p53 target gene whose loss produces a similar apoptotic defect to p53 loss in irradiated T lymphocytes (Jeffers et al. 
2003). These data suggest that PUMA is an essential p53 effector during apoptosis_-at least in this cell type under these conditions. However, PUMA-null mice are not overtly tumor prone, thus suggesting that simultaneous inactivation of multiple p53 effector functions is critical for initiating tumorigenesis. This simple example illustrates the complex nature of the p53 network, and highlights the importance of understanding context-dependent functions of p53 (discussed in the following section).

\section{Which p53 Effector Functions Are Crucial for Tumor Suppression?}

The aforementioned section describes several processes that might contribute to p53 tumor suppressor activity; thereby raising the question of which ones (or one) are crucial. Studies to address this question have achieved apparently contradictory results. Most studies have approached this question by phenocopying p53 loss through either the disruption of p53 target genes specific to particular p53 effector functions or the use of separation-of-function p53 mutants, and studying the resulting biological impact. For example, in lymphoma, the impact of $\mathrm{p} 53$ on myc-induced lymphomagenesis was compared with defects in apoptosis; for example, Bcl-2 overexpression or inactivation of PUMA (Schmitt et al. 2002a; Hemann et al. 2004). Results in this setting suggest that disruption of apoptosis is sufficient to explain p53 tumor suppressor function. However, PUMA null mice or Bcl-2 transgenics are not as tumor prone as p53 null animals, suggesting to some that apoptosis alone is not a crucial function of p53.

The interpretation of studies comparing the biological effects of p53 loss to the biological effects of loss of a key p53 effector has numerous limitations. For example, they necessarily assume that a particular p53 effector controls one p53 effector function. However, this may not always be the case-for example, p21 Waf1/Cip1 is a p53 effector involved in growth arrest but it also acts, in some settings, to attenuate apoptosis (Seoane et al. 2002). Moreover, the miR34 microRNA family can influence both cellcycle arrest and apoptosis (He et al. 2007).
Additionally, studies that rely on knock-out mouse are prone to developmental compensation, particularly in situations in which they examine one member of a larger gene family (e.g., Bax and Bak). Finally, just because deletion of one gene (e.g., PUMA) member creates a phenotype that approaches p53 loss does not necessarily indicate that other p53 effectors are NOT important. Hence, if multiple effectors coordinate the same process together-as PUMA requires Bax for apoptosis (Letai 2009) - then deletion of more than one effector could give near complete phenotypes.

Several studies have used p53 mutant alleles that separate $\mathrm{p} 53$ functions to query the contribution of different p53 effector functions to p53-mediated tumor suppression. One p53 mutant (containing the amino acid substitution R175P) is completely defective at inducing apoptosis, but can still induce cell-cycle arrest (Liu et al. 2004a). Transgenic mice expressing this p53 mutant lived longer and developed significantly fewer tumors compared to p53 null mice. Additionally, the tumors that eventually developed retained a diploid chromosome number, in stark contrast to the aneuploidy observed in tumors from p53 null mice. These data suggest that p53-dependent apoptosis is dispensable for tumor suppression, whereas the ability of p53 to maintain genetic stability is what is important for tumor suppression.

In several other studies, the contribution of the transcriptional activation function of p53 to p53-effector functions was probed. In one study mentioned earlier, a mutant allele of p53 ( $\mathrm{p} 53 \mathrm{Q}$ ) encoding a protein with mutations in the transactivation domain of p53 was used to generate transgenic mice (Johnson et al. 2005). Although the ability of p53QS to transactivate p53 target genes was largely compromised, it retained the ability to transactivate the apoptotic target Bax. This, however, was insufficient for p53QS to induce apoptosis in response to DNA damage. Interestingly, p53QS was able to partially induce apoptosis in response to serum deprivation, and maintained a robust apoptotic response on exposure to hypoxia.

In a complementary study, a chimeric p53 protein was generated that was fully capable 
of transactivation ( $533 \mathrm{VP} 16)$, but lacking domains involved in transactivation-independent functions (i.e., transcriptional repression) (Johnson et al. 2008). p53VP16 was able to robustly transactivate p53 targets involved both in apoptosis and senescence. However, p53VP16 was only able to induce senescence, and not apoptosis, under a variety of conditions in mouse fibroblasts. Taken together, these studies show a role of p53 transactivation in senescence, at the same time suggesting that transactivation is insufficient for apoptosis, at least in certain contexts.

Studies using such p53 mutants also have limitations. Although they depend in large part on the mutants being true "separation-of-function" alleles, this is unlikely to be the case. Also, mutations that affect the p53-Mdm2 interaction may compromise transcription but dramatically elevate p53 levels, such that cells with wild-type and mutant p53 have different p53 levels.

Although it is formally possible that technical issues might explain the apparent discrepancies described earlier, it is also possible that the key p53 effector functions are context dependent. Two examples from our group highlight this in great detail. First, in the $\mathrm{E} \mu$-myc lymphoma model, disruption of apoptosis is sufficient to phenocopy p53 loss during tumorigenesis but not following cytotoxic cancer therapy, in which disabling p53-dependent senescence was also required (Schmitt et al. 2002b). Moreover, in a cell culture transformation model, disruption of the apoptosis effector PUMA mimicked p53 loss in cooperating with myc and not ras (Hemann et al. 2004).

In support of aforementioned findings, numerous studies on the reactivation of p53 in various tumors reveal the context dependence of p53 function. Using different strategies, three independent groups have examined the consequences of reactivating endogenous p53 in p53-deficient tumors in mice. In one study, a Cre-loxP-based strategy was used to temporally control p53 expression in vivo (Ventura et al. 2007). This study reported that restoration of endogenous p53 leads to the regression of lymphomas and sarcomas without affecting normal tissue. Importantly, the mechanism of tumor regression was dependent on tumor type, i.e., lymphoma regressed via apoptosis, whereas sarcomas regressed primarily via cellular senescence. In another study, the p53 gene was replaced by a gene encoding a p53 fusion protein whose function is completely dependent on the presence of 4-hydroxytamoxifen (4-OHT) (Christophorou et al. 2005). In this mouse model, addition of 4-OHT (to generate wild-type p53) in the presence of ionizing radiation resulted in a potent $\mathrm{p} 53$-dependent apoptotic response in the thymus and spleen. In contrast, although consistent with p53 action, addition of 4-OHT to MEFs cooperated with expression of the activated ras oncogene to induce a senescence-like arrest. Similar data were reported through the utilization of an inducible RNAi-based strategy to reactivate p53 in in vitro and in vivo (Dickins et al. 2005; Dickins et al. 2007). Finally, as discussed previously, reactivation of endogenous p53 using reversible RNAi in liver carcinoma leads to senescence and, ultimately, tumor clearance by recruitment of the innate immune system (Xue et al. 2007). Hence, as suggested from cell culture studies, the effects of p53 induction in vivo are dependent on cell type and genetic background.

\section{UNDERSTANDING CONTEXT DEPENDENCE}

The findings that different p53 effector functions can be important for tumor suppression implies that p53 action is context dependent. How and why this is remains an active area of investigation. It seems likely that such context dependence is affected by the cell type, the genetic background of the cell, the microenvironment of the cell, and the nature of the stress placed on the cell. As previously noted, in response to DNA damage, some cell types undergo p53dependent apoptosis (T lymphocytes), whereas other cells undergo growth arrest (MEFs). The mechanisms underlying such differences are still not clear, although it is possible that other factors make the apoptotic machinery more "accessible" in thymocytes, which are programmed to respond to a variety of stimuli by apoptosis. 
The genetic changes acquired in particular cells are equally important contributors to context dependence. For example, it was previously mentioned that MEFs exposed to DNA damage undergo p53-dependent growth arrest, and this is mediated by the p53-mediated up-regulation of $p 21^{\text {Waf1/Cip } 1}$. Gene expression analysis of these MEFs reveals that numerous other p53 targets, such as PUMA and Bax, are also up-regulated, yet these cells do not undergo apoptosis. However, introducing a single gene into these MEFs, such as the myc or E1A oncogenes, can "rewire" these cells to become predisposed to p53-dependent apoptosis rather than growth arrest. Conversely, the introduction of the RAS oncogene into MEFs, independent of DNA damage, results in p53dependent senescence. In all circumstances, numerous p53 target genes, involved in different biological processes, are up-regulated, but the cells respond differently-be it transient cellcycle arrest, apoptosis, or senescence.

The aforementioned data suggest that it is imperative when identifying and/or elucidating the function of a p53 effector gene, that it is studied in the appropriate context. This context dependence was also shown with the p53 apoptotic target gene PUMA as a tumor suppressor gene, only when studied in the appropriate context (Hemann et al. 2004). Using RNA interference to evaluate PUMA as a tumor suppressor, we found suppression of PUMA can approximate the effects of p53 loss during E1A/Ras-mediated transformation of primary MEFs and during myc-induced lymphomagenesis. Importantly, these data must be evaluated in light of the context of the experiments, for example, E1A/Ras MEFs and myc-induced lymphomagenesis both "rewire" the cells to undergo p53-mediated apoptosis. Acute suppression of PUMA expression, using a short hairpin RNA targeting PUMA (shPUMA), in these cells is equivalent to loss of p53 with respect to tumor formation. However, unlike the lymphomas arising from the suppression of $\mathrm{p} 53$, the lymphomas arising from suppression of PUMA still maintained cell-cycle checkpoints. This is consistent with PUMA being exclusively involved in apoptosis and the cell-cycle checkpoint. Whereas, because p53 regulates both cell cycle and apoptosis, loss of p53 results in an abrogation of both functions.

As previously discussed, introduction of E1A/Ras into MEFs results in p53-dependent apoptosis, whereas the introduction of only Ras results in p53-mediated permanent growth arrest. Consistently, suppression of p53 expression in either of these contexts results in the transformation of these MEFs. Significantly, only E1A/Ras, and not Ras, cooperated with PUMA suppression to transform these MEFs. These data show that PUMA can function as a tumor suppressor in the context of a p53dependent apoptosis setting. Interestingly, the lack of tumors found in PUMA null mice may appear, on its face, as contradictory to the conclusion that PUMA is a tumor suppressor. However, these data may actually expand our definition of "tumor suppressor" as the inclusion of context dependence is crucial.

\section{Mechanisms of Context Dependence}

The precise mechanism underlying the context dependence of p53 function remains mysterious. However, understanding the underpinnings of this context dependence will reveal the critical nodes of the p53 network in selected cellular settings, and consequently allow for more selective manipulation of p53 biological functions in cancer, aging, and other diseases. Two nonexclusive models have been put forward to explain the context-dependent nature of p53 function (Vousden 2000), as discussed and expanded on later.

The first model suggests that the qualitative and/or quantitative changes to p53 directly impact its biological output. In this model, p53 functions differently in cells that will undergo cell-cycle arrest than in cells destined to undergo apoptosis, and the amount of p53 or the presence of different forms can activate cell-cycle arrest or apoptotic target genes. For example, it has previously been shown that low levels of p53 can lead to cell-cycle arrest, whereas increasing levels can trigger apoptosis in the human cancer cell lines (Chen et al. 
1996). Although this finding may be cell-type specific, it is possible that the promoters for p53 target genes involved in cell-cycle arrest have a higher affinity for p53 than promoters of apoptotic genes. Additionally, the binding of p53 to these promoters may be qualitatively regulated by the posttranslational modifications of p53. For example, the phosphorylation of p53 at serine 46 has been shown to correlate with apoptosis, and may be partially explained by the ability of this phosphorylation to regulate expression of at least one p53 apoptotic target, p53AIP1 (Oda et al. 2000). Numerous posttranslational modifications of p53 at various amino acids have been described, including phosphorylation, ubiquitination, acetylation, methylation, sumoylation, neddylation, glycosylation, and ribosylation (Carter and Vousden 2009; Kruse and $\mathrm{Gu} 2009)$.

The model in which the qualitative and/or quantitative changes to p53 can impact its output predicts that distinct target genes are induced under different circumstances. Clearly, this is not always the case. As previously mentioned, in cells destined to undergo apoptosis, p53 targets involved in cell-cycle arrest are also activated. This can be partially explained by a second model where the cell interprets p53 signals differently depending on what other genes are expressed in the cell. In this view, the degree of overlap between the p53 signaling network and other signaling networks determines the output. Although this model has not been conclusively shown, the general concept can be illustrated through a hypothetical example involving microRNAs. The p53 target microRNA, miR-34, can induce apoptosis or senescence in different cell types (He et al. 2007). In this scenario, p53 is inducing the same microRNA, but the biological outcome is different. Although it has yet to be shown, it is obvious that miR-34 will only act on target genes (i.e., genes with seed sequences in the $3^{\prime} \mathrm{UTR}$ ) that are expressed. Consequently, if these target genes are predominantly growth promoting genes, then the outcome will be arrest, and if they are predominantly cell survival genes, the outcome will be apoptosis. It seems likely that cross talk between the PI3 kinase pathway and the NF-kB pathway can also have an impact on p53 effector functions.

The model suggesting that it is the interplay between p53 signaling and other activated signaling pathways in the cell that dictates the biological outcome is additionally reinforced by the findings that PUMA is a "significant" p53 effector in the presence of myc and E1A, but not ras (previously discussed). When PUMA is ablated in the presence of E1A or myc (in which the output is apoptosis), tumors form. In contrast, ablation of PUMA in the presence of ras (in which the output is senescence) has no effect on tumorigenesis. This may be explained by the presence of parallel pathways, whereby myc and E1A block growth arrest, whereas ras blocks apoptosis, so the remaining process is the predominant output of p53.

\section{CONCLUSION}

As a testament to the significance and complexity of the p53 network, there are over 50,000 papers published relating to the biochemical and biological properties of p53. Consequently, there is an ever-growing list of p53 targets and effector functions. The next imperative duty is to unravel the mechanisms that dictate the biological outputs of the p53 network, in other words, understanding the context dependent functions of $\mathrm{p} 53$. As discussed throughout this article, this task requires consideration of p53 itself (modification and localization of the protein), its activated and repressed targets, the genetic background of the cell, and the extracellular environment. A better understanding of the context dependent biological output of the p53 network should help in predicting disease progression and response, and in treating cancers, aging, and other disorders.

\section{ACKNOWLEDGMENTS}

This work was supported in part by a program project grant from the National Cancer Institute and the Don Monti Memorial Research Foundation. S.W.L. is an investigator in the Howard Hughes Medical Institute. 


\section{REFERENCES}

Abida WM, Gu W. 2008. p53-Dependent and p53-independent activation of autophagy by ARF. Cancer Res 68: 352-357.

Appella E, Anderson CW. 2001. Post-translational modifications and activation of p53 by genotoxic stresses. Eur J Biochem 268: 2764-2772.

Brown JP, Wei W, Sedivy JM. 1997. Bypass of senescence after disruption of p21CIP1/WAF1 gene in normal diploid human fibroblasts. Science 277: 831-834.

Brugarolas J, Chandrasekaran C, Gordon JI, Beach D, Jacks T, Hannon GJ. 1995. Radiation-induced cell cycle arrest compromised by p21 deficiency. Nature 377: 552-557.

Campisi J, d'Adda di Fagagna F. 2007. Cellular senescence: when bad things happen to good cells. Nat Rev Mol Cell Biol 8: 729-740.

Carter S, Vousden KH. 2009. Modifications of p53: competing for the lysines. Curr Opin Genet Dev 19: 18-24.

Chan HM, La Thangue NB. 2001. p300/CBP proteins: HATs for transcriptional bridges and scaffolds. J Cell Sci 114: 2363-2373.

Chan TA, Hermeking H, Lengauer C, Kinzler KW, Vogelstein B. 1999. 14-3-3Sigma is required to prevent mitotic catastrophe after DNA damage. Nature 401: 616-620.

Chen X, Ko LJ, Jayaraman L, Prives C. 1996. p53 levels, functional domains, and DNA damage determine the extent of the apoptotic response of tumor cells. Genes Dev 10: 2438-2451.

Chipuk JE, Bouchier-Hayes L, Kuwana T, Newmeyer DD, Green DR. 2005. PUMA couples the nuclear and cytoplasmic proapoptotic function of p53. Science 309: $1732-1735$.

Christophorou MA, Martin-Zanca D, Soucek L, Lawlor ER, Brown-Swigart L, Verschuren EW, Evan GI. 2005. Temporal dissection of p53 function in vitro and in vivo. Nat Genet 37: 718-726.

Clarke AR, Purdie CA, Harrison DJ, Morris RG, Bird CC Hooper ML, Wyllie AH. 1993. Thymocyte apoptosis induced by p53-dependent and independent pathways. Nature 362: 849-852.

Crighton D, O'Prey J, Bell HS, Ryan KM. 2007. p73 regulates DRAM-independent autophagy that does not contribute to programmed cell death. Cell Death Differ 14: 1071-1079.

Crighton D, Wilkinson S, O'Prey J, Syed N, Smith P, Harrison PR, Gasco M, Garrone O, Crook T, Ryan KM. 2006. DRAM, a p53-induced modulator of autophagy, is critical for apoptosis. Cell 126: 121-134.

de Rozieres S, Maya R, Oren M, Lozano G. 2000. The loss of mdm2 induces p53-mediated apoptosis. Oncogene 19: 1691-1697.

Deng C, Zhang P, Harper JW, Elledge SJ, Leder P. 1995. Mice lacking p21CIP1/WAF1 undergo normal development, but are defective in G1 checkpoint control. Cell 82: 675-684.

Dickins RA, Hemann MT, Zilfou JT, Simpson DR, Ibarra I, Hannon GJ, Lowe SW. 2005. Probing tumor phenotypes using stable and regulated synthetic microRNA precursors. Nat Genet 37: 1289-1295.
Dickins RA, McJunkin K, Hernando E, Premsrirut PK, Krizhanovsky V, Burgess DJ, Kim SY, Cordon-Cardo C, Zender L, Hannon GJ, et al. 2007. Tissue-specific and reversible RNA interference in transgenic mice. Nat Genet 39: 914-921.

Eisenberg-Lerner A, Kimchi A. 2009. The paradox of autophagy and its implication in cancer etiology and therapy. Apoptosis 14: 376-391.

el-Deiry WS, Kern SE, Pietenpol JA, Kinzler KW, Vogelstein B. 1992. Definition of a consensus binding site for p53. Nat Genet 1: 45-49.

Espinosa JM, Verdun RE, Emerson BM. 2003. p53 functions through stress- and promoter-specific recruitment of transcription initiation components before and after DNA damage. Mol Cell 12: 1015-1027.

Fridman JS, Lowe SW. 2003. Control of apoptosis by p53. Oncogene 22: 9030-9040.

Giono LE, Manfredi JJ. 2006. The p53 tumor suppressor participates in multiple cell cycle checkpoints. $J$ Cell Physiol 209: 13-20.

Gorgoulis VG, Zacharatos P, Kotsinas A, Kletsas D, Mariatos G, Zoumpourlis V, Ryan KM, Kittas C, Papavassiliou AG. 2003. p53 activates ICAM-1 (CD54) expression in an NF-кB-independent manner. Embo J 22: 1567-1578.

Green DR, Kroemer G. 2009. Cytoplasmic functions of the tumour suppressor p53. Nature 458: 1127-1130.

Hainaut P, Hollstein M. 2000. p53 and human cancer: the first ten thousand mutations. Adv Cancer Res 77: 81-137.

Hayflick L. 1965. The Limited in Vitro Lifetime of Human Diploid Cell Strains. Exp Cell Res 37: 614-636.

He L, He X, Lim LP, de Stanchina E, Xuan Z, Liang Y, Xue W Zender L, Magnus J, Ridzon D, et al. 2007. A microRNA component of the p53 tumour suppressor network. Nature 447: 1130-1134.

Hemann MT, Zilfou JT, Zhao Z, Burgess DJ, Hannon GJ, Lowe SW. 2004. Suppression of tumorigenesis by the p53 target PUMA. Proc Natl Acad Sci 101: 9333-9338.

Hermeking H, Lengauer C, Polyak K, He TC, Zhang L, Thiagalingam S, Kinzler KW, Vogelstein B. 1997. 14-3-3 sigma is a p53-regulated inhibitor of G2/M progression. Mol Cell 1: 3-11.

Humbey O, Pimkina J, Zilfou JT, Jarnik M, DominguezBrauer C, Burgess DJ, Eischen CM, Murphy ME. 2008. The ARF tumor suppressor can promote the progression of some tumors. Cancer Res 68: 9608-9613.

Iyer NG, Ozdag H, Caldas C. 2004. p300/CBP and cancer. Oncogene 23: 4225-4231.

Jeffers JR, Parganas E, Lee Y, Yang C, Wang J, Brennan J, MacLean KH, Han J, Chittenden T, Ihle JN, et al. 2003. Puma is an essential mediator of p53-dependent and -independent apoptotic pathways. Cancer Cell 4: 321-328.

Johnson TM, Hammond EM, Giaccia A, Attardi LD. 2005. The p53QS transactivation-deficient mutant shows stress-specific apoptotic activity and induces embryonic lethality. Nat Genet 37: 145-152.

Johnson TM, Meade K, Pathak N, Marques MR, Attardi LD. 2008. Knockin mice expressing a chimeric p53 protein reveal mechanistic differences in how p53 triggers apoptosis and senescence. Proc Natl Acad Sci 105: $1215-$ 1220. 


\section{J.T. Zilfou and S.W. Lowe}

Kaplowitz N. 2001. Drug-induced liver disorders: implications for drug development and regulation. Drug Saf 24: $483-490$.

Krizhanovsky V, Xue W, Zender L, Yon M, Hernando E, Lowe SW. 2008a. Implications of cellular senescence in tissue damage response, tumor suppression, and stem cell biology. Cold Spring Harb Symp Quant Biol 73: 513-522.

Krizhanovsky V, Yon M, Dickins RA, Hearn S, Simon J, Miething C, Yee H, Zender L, Lowe SW. 2008b. Senescence of activated stellate cells limits liver fibrosis. Cell 134: 657-667.

Kruse JP, Gu W. 2009. Modes of p53 regulation. Cell 137: 609-622.

Lane DP. 1992. Cancer. p53, guardian of the genome. Nature 358: $15-16$.

Letai A. 2009. Puma strikes Bax. J Cell Biol 185: 189-191.

Levine AJ. 1997. p53, the cellular gatekeeper for growth and division. Cell 88: 323-331.

Liang XH, Jackson S, Seaman M, Brown K, Kempkes B, Hibshoosh H, Levine B. 1999. Induction of autophagy and inhibition of tumorigenesis by beclin 1. Nature 402: $672-676$.

Liu Y, Lagowski JP, Vanderbeek GE, Kulesz-Martin MF. 2004b. Facilitated search for specific genomic targets by p53 C-terminal basic DNA binding domain. Cancer Biol Ther 3: 1102-1108.

Liu G, Parant JM, Lang G, Chau P, Chavez-Reyes A, El-Naggar AK, Multani A, Chang S, Lozano G. 2004a. Chromosome stability, in the absence of apoptosis, is critical for suppression of tumorigenesis in Trp53 mutant mice. Nat Genet 36: 63-68.

Lowe SW, Ruley HE. 1993. Stabilization of the p53 tumor suppressor is induced by adenovirus 5 E1A and accompanies apoptosis. Genes Dev 7: 535-545.

Lowe SW, Schmitt EM, Smith SW, Osborne BA, Jacks T. 1993. p53 is required for radiation-induced apoptosis in mouse thymocytes. Nature 362: 847-849.

Montes de Oca Luna R, Wagner DS, Lozano G. 1995. Rescue of early embryonic lethality in mdm2-deficient mice by deletion of p53. Nature 378: 203-206.

Murphy ME. 2003. The thousand doors that lead to death: p53-dependent repression and apoptosis. Cancer Biol Ther 2: 381-382.

Narita M, Narita M, Krizhanovsky V, Nunez S, Chicas A, Hearn SA, Myers MP, Lowe SW. 2006. A novel role for high-mobility group a proteins in cellular senescence and heterochromatin formation. Cell 126: 503-514.

Narita M, Nunez S, Heard E, Narita M, Lin AW, Hearn SA, Spector DL, Hannon GJ, Lowe SW. 2003. Rb-mediated heterochromatin formation and silencing of E2F target genes during cellular senescence. Cell 113:703-716.

Oda K, Arakawa H, Tanaka T, Matsuda K, Tanikawa C, Mori T, Nishimori H, Tamai K, Tokino T, Nakamura Y, Taya Y. 2000. p53AIP1, a potential mediator of p53-dependent apoptosis, and its regulation by Ser-46-phosphorylated p53. Cell 102: 849-862.

Parant J, Chavez-Reyes A, Little NA, Yan W, Reinke V, Jochemsen AG, Lozano G. 2001. Rescue of embryonic lethality in Mdm4-null mice by loss of Trp53 suggests a nonoverlapping pathway with MDM2 to regulate p53. Nat Genet 29: 92-95.

Pimkina J, Humbey O, Zilfou JT, Jarnik M, Murphy ME. 2009. ARF induces autophagy by virtue of interaction with Bcl-xl. J Biol Chem 284: 2803-2810.

Riley T, Sontag E, Chen P, Levine A. 2008. Transcriptional control of human p53-regulated genes. Nat Rev Mol Cell Biol 9: 402-412.

Roninson IB. 2003. Tumor cell senescence in cancer treatment. Cancer Res 63: 2705-2715.

Schmitt CA, Fridman JS, Yang M, Baranov E, Hoffman RM, Lowe SW. 2002a. Dissecting p53 tumor suppressor functions in vivo. Cancer Cell 1: 289-298.

Schmitt CA, Fridman JS, Yang M, Lee S, Baranov E, Hoffman RM, Lowe SW. 2002b. A senescence program controlled by p53 and p16INK4a contributes to the outcome of cancer therapy. Cell 109: 335-346.

Seoane J, Le HV, Massague J. 2002. Myc suppression of the p21(Cip1) Cdk inhibitor influences the outcome of the p53 response to DNA damage. Nature 419: 729-734.

Serrano M, Lin AW, McCurrach ME, Beach D, Lowe SW. 1997. Oncogenic ras provokes premature cell senescence associated with accumulation of p53 and p16INK4a. Cell 88: 593-602.

Sherr CJ. 2006. Divorcing ARF and p53: an unsettled case. Nat Rev Cancer 6: 663-673.

St Clair S, Manfredi JJ. 2006. The dual specificity phosphatase $\mathrm{Cdc} 25 \mathrm{C}$ is a direct target for transcriptional repression by the tumor suppressor p53. Cell Cycle 5: 709-713.

Stewart SA, Weinberg RA. 2006. Telomeres: cancer to human aging. Annu Rev Cell Dev Biol 22: 531-557.

Tasdemir E, Maiuri MC, Galluzzi L, Vitale I, DjavaheriMergny M, D’Amelio M, Criollo A, Morselli E, Zhu C, Harper F, et al. 2008. Regulation of autophagy by cytoplasmic p53. Nat Cell Biol 10: 676-687.

Ventura A, Kirsch DG, McLaughlin ME, Tuveson DA, Grimm J, Lintault L, Newman J, Reczek EE, Weissleder R, Jacks T. 2007. Restoration of p53 function leads to tumour regression in vivo. Nature 445: 661-665.

Vousden KH. 2000. p53: death star. Cell 103: 691-694.

Xue W, Zender L, Miething C, Dickins RA, Hernando E, Krizhanovsky V, Cordon-Cardo C, Lowe SW. 2007. Senescence and tumour clearance is triggered by p53 restoration in murine liver carcinomas. Nature 445: 656-660.

Yee KS, Vousden KH. 2005. Complicating the complexity of p53. Carcinogenesis 26: 1317-1322.

Young AR, Narita M, Ferreira M, Kirschner K, Sadaie M, Darot JF, Tavare S, Arakawa S, Shimizu S, Watt FM, et al. 2009. Autophagy mediates the mitotic senescence transition. Genes Dev 23: 798-803.

Yue Z, Jin S, Yang C, Levine AJ, Heintz N. 2003. Beclin 1, an autophagy gene essential for early embryonic development, is a haploinsufficient tumor suppressor. Proc Natl Acad Sci 100: 15077-15082.

Zilfou JT, Hoffman WH, Sank M, George DL, Murphy M. 2001. The Corepressor mSin3a Interacts with the Proline-Rich Domain of p53 and Protects p53 from Proteasome-Mediated Degradation. Mol Cell Biol 21: 3974-3985. 


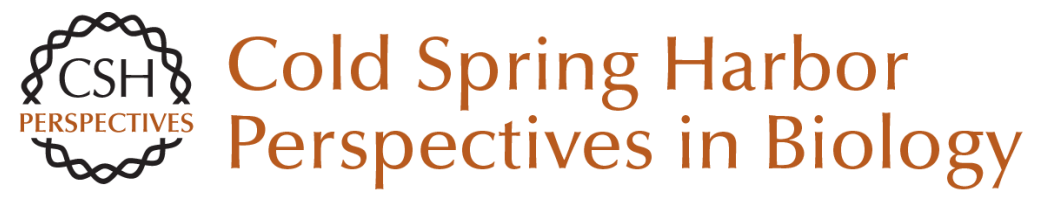

\section{Tumor Suppressive Functions of p53}

Jack T. Zilfou and Scott W. Lowe

Cold Spring Harb Perspect Biol 2009; doi: 10.1101/cshperspect.a001883 originally published online October 21, 2009

\section{Subject Collection The p53 Family}

The Origins and Evolution of the p53 Family of Genes

Vladimir A. Belyi, Prashanth Ak, Elke Markert, et al.

Mouse Models of p53 Functions

Guillermina Lozano

TP53 Mutations in Human Cancers: Origins,

Consequences, and Clinical Use

Magali Olivier, Monica Hollstein and Pierre Hainaut

p53 Research: The Past Thirty Years and the Next

Thirty Years

David Lane and Arnold Levine

Transcriptional Regulation by P53

Rachel Beckerman and Carol Prives

p53-based Cancer Therapy

David P. Lane, Chit Fang Cheok and Sonia Lain

Phylogeny and Function of the Invertebrate p53

Superfamily

Rachael Rutkowski, Kay Hofmann and Anton Gartner

Tied Up in Loops: Positive and Negative

Autoregulation of p53

Xin Lu
The Tumor Suppressor p53: From Structures to

Drug Discovery

Andreas C. Joerger and Alan R. Fersht

p53 Regulation of Metabolic Pathways

Eyal Gottlieb and Karen H. Vousden

The Regulation of the p53-mediated Stress

Response by MDM2 and MDM4 Mary Ellen Perry

Zebrafish Models of p53 Functions Narie Y. Storer and Leonard I. Zon

p63 and p73, the Ancestors of p53

V. Dötsch, F. Bernassola, D. Coutandin, et al.

Pathologies Associated with the p53 Response Andrei V. Gudkov and Elena A. Komarova

Single-nucleotide Polymorphisms in the p53

Signaling Pathway

Lukasz F. Grochola, Jorge Zeron-Medina, Sophie Mériaux, et al.

Clinical Outcomes and Correlates of TP53

Mutations and Cancer

Ana I. Robles and Curtis C. Harris

For additional articles in this collection, see http://cshperspectives.cshlp.org/cgi/collection/

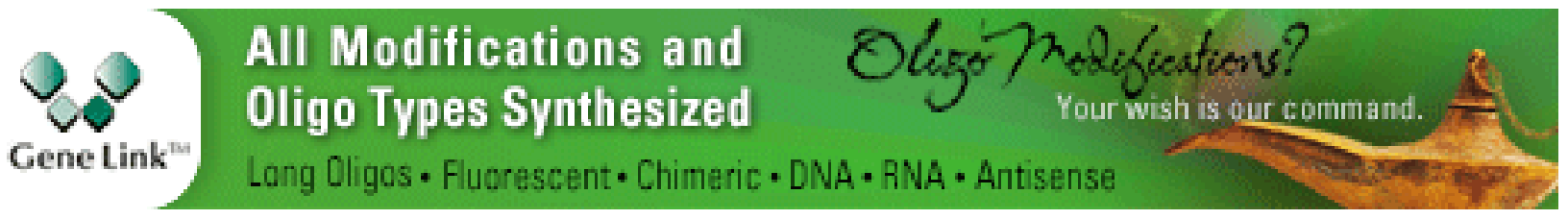

Copyright @ 2009 Cold Spring Harbor Laboratory Press; all rights reserved 